\title{
Complex Period Variations of the Neglected W UMa-type Binary System NY Lyrae
}

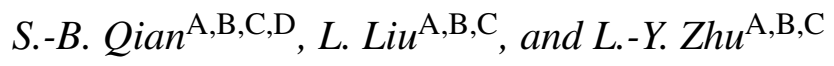 \\ A National Astronomical Observatories/Yunnan Observatory, Chinese Academy of Sciences, \\ P.O. Box 110, 650011 Kunming, P.R. China \\ B United Laboratory of Optical Astronomy, Chinese Academy of Sciences, \\ 100012 Beijing, P.R. China \\ ${ }^{\mathrm{C}}$ Graduate School of the CAS, Beijing, P.R. China \\ D Corresponding author. Email: qsb@ynao.ac.cn
}

Received 2008 June 3, accepted 2008 September 30

\begin{abstract}
Orbital-period variations of the neglected W UMa-type binary star, NY Lyr, were analyzed based on two newly determined eclipse times together with the others compiled from the literature. A cyclic oscillation with a period of $82.1 \mathrm{yr}$ and an amplitude of $0.0247 \mathrm{~d}$ was discovered to be superimposed on a continuous period increase $\left(\mathrm{d} P / \mathrm{d} t=+1.33 \times 10^{-7} \mathrm{~d} \mathrm{yr}^{-1}\right)$. After the long-term period increase and the large-amplitude cyclic oscillation were removed from the $\mathrm{O}-\mathrm{C}$ diagram, the residuals suggest that there is another smallamplitude period oscillation $\left(A_{4}=0.0053 \mathrm{~d}, P_{4}=19.4\right.$ years $)$ in the orbital period changes. As in the cases of $\mathrm{AH} \mathrm{Cnc}$ and AD Cnc, both the continuous period increase and the two cyclic period oscillations make NY Lyr an interesting system to study in the future. In order to understand the evolutionary state of the binary system, new photometric and spectroscopic observations and a careful investigation on those data are needed.
\end{abstract}

Keywords: stars: binaries: close — stars: binaries: eclipsing — stars: individual (NY Lyrae)

\section{Introduction}

NY Lyr (VV No. 133, AN 214.1935 and GSC 2661.00727) is a neglected close-binary system. Up to now, only 13 papers related to the system were listed in ADS (Astrophysics Data System), and most of them published times of light minimum of the binary. According to the 4th edition of the general catalogue of variable stars (GCVS) (Kholopov et al. 1985), it is an EW/KW-type binary star with a short period of $0.44079534 \mathrm{~d}$. The magnitude range of the eclipsing binary is from 12.7 to $13.2 \mathrm{mag}$. The light variability of the binary was discovered by Morgenroth (1935). It is well known that orbital period changes of W UMa-type binaries are very common (e.g. Qian 2001a,b, 2003). For NY Lyr, the O-C diagram published by Kreiner et al. (2001) suggested that its orbital period is changeable. In order to study the properties of the period variation of the eclipsing binary, it was included in our photometric monitor for times of light minimum.

\section{New CCD Data for NY Lyr}

The observational data of NY Lyr were carried out on April 25 and July 26, 2006 with the PI1024 TKB CCD photometric system attached to the $1.0-\mathrm{m}$ reflect telescope at the Yunnan Observatory in China. During the observation, $V$ and $R$ filters that are close to the standard Johnson UBVRI system (Yang \& Li 1999) were used. The integration time for each image is $90 \mathrm{~s}$. Two stars in the same field of view of NY Lyr were chosen as the comparison and
Table 1. Coordinates and magnitudes of NY Lyr: comparison and check stars

\begin{tabular}{lccc}
\hline Stars & $\alpha_{2000}$ & $\delta_{2000}$ & $V$ \\
\hline NY Lyr & $19^{\mathrm{h}} 16^{\mathrm{m}} 36.9^{\mathrm{s}}$ & $34^{\circ} 23^{\prime} 40.0^{\prime \prime}$ & 11.9 \\
Comparison & $19^{\mathrm{h}} 16^{\mathrm{m}} 39.4^{\mathrm{s}}$ & $34^{\circ} 23^{\prime} 04.4^{\prime \prime}$ & 11.7 \\
Check & $19^{\mathrm{h}} 16^{\mathrm{m}} 39.9^{\mathrm{s}}$ & $34^{\circ} 23^{\prime} 22.4^{\prime \prime}$ & 12.2 \\
\hline
\end{tabular}

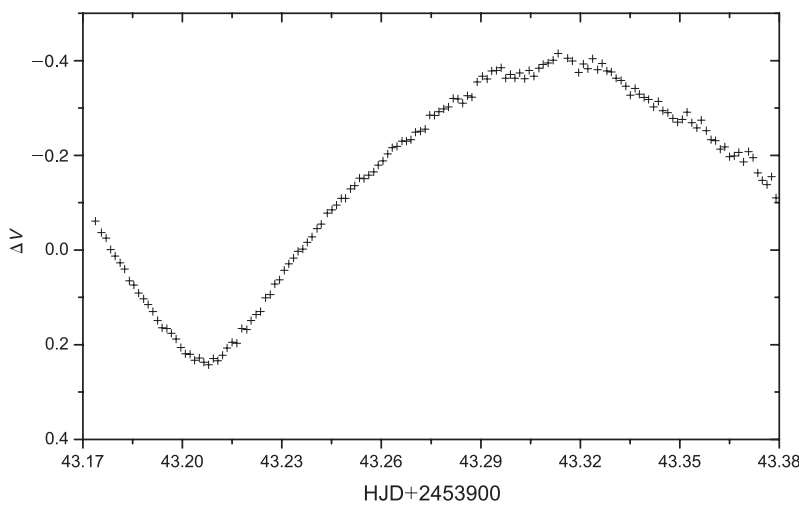

Figure 1 CCD observations in $V$ for NY Lyr observed on 2006 July 26.

check stars. Their coordinates and magnitudes are listed in Table 1. Рнот (measure magnitudes for a list of stars) of the aperture photometry package of the IRAF was used to reduce the observed images. The observations obtained 
on July 26, 2006 are plotted in Figure 1. It is shown in this figure the light variation is typical EW type where the light changes continuously. Although complete light curves were not obtained, two times of light minimum were derived from the observations by using a parabolic fitting method and they are listed in Table 2. Since the light curve shows slightly asymmetric, during the determination of the eclipse times, only the lower portion of the minimum was used.

Table 2. New CCD times of light minimum for NY Lyr

\begin{tabular}{lccc}
\hline $\begin{array}{l}\text { J.D. (Hel.) } \\
\text { (days) }\end{array}$ & $\begin{array}{c}\text { Error } \\
\text { (days) }\end{array}$ & Min. & Filter \\
\hline 2453851.2977 & \pm 0.0004 & II & R \\
2453943.2069 & \pm 0.0002 & I & V \\
\hline
\end{tabular}

\section{Orbital-Period Changes for NY Lyr}

All available times of light minimum of NY Lyr are displayed in Table 3. The information in the second column of the table refers to the observed methods where 'pg' and 'vis' refer to photographic and visual measurements, respectively, 'pe' to photoelectric data and 'CCD' to charge-couple device. Although times of light minimum of the W UMa-type binary NY Lyr were published by many authors (see in Table 3), it was neglected for orbital period study. The $(\mathrm{O}-\mathrm{C})_{1}$ values were calculated with the linear ephemeris given by Kreiner et al. (2001),

$$
H J D_{\mathrm{I}_{\min }}=\mathrm{HJD} 2445196.4188+0.4407942 E .
$$

The corresponding $(\mathrm{O}-\mathrm{C})_{1}$ diagram is shown in the upper panel of Figure 2 where open circles refer to photographic or visual times of light minimum and solid dots to photoelectric data and CCD observations.

Table 3. Times of light minimum for NY Lyr

\begin{tabular}{|c|c|c|c|c|c|c|c|c|}
\hline $\mathrm{HJD}(+2400000)$ (days) & Methods & Min. & $E$ & $(\mathrm{O}-\mathrm{C})_{1}$ (days) & $(\mathrm{O}-\mathrm{C})_{2}$ (days) & $(\mathrm{O}-\mathrm{C})_{3}$ (days) & Res. (days) & Ref. $^{\mathrm{a}}$ \\
\hline 15253.3720 & pg & I & -67930.0 & +0.1032 & -0.0308 & -0.0082 & & (1) \\
\hline 18287.1193 & pg & II & -61047.5 & +0.0844 & -0.0018 & +0.0103 & & (1) \\
\hline 27902.7529 & pg & I & -39233.0 & +0.0129 & +0.0278 & +0.0032 & & (1) \\
\hline 28749.9455 & pg & I & -37311.0 & -0.0009 & +0.0193 & -0.0052 & & (1) \\
\hline 30606.3453 & pg & II & -33099.5 & -0.0058 & +0.0239 & +0.0022 & & (1) \\
\hline 34162.2151 & pg & II & -25032.5 & -0.0229 & +0.0170 & +0.0090 & & (1) \\
\hline 34714.0831 & $\mathrm{pg}$ & II & -23780.5 & -0.0292 & +0.0113 & +0.0061 & & (1) \\
\hline 34991.7723 & pg & II & -23150.5 & -0.0404 & +0.0003 & -0.0034 & & (1) \\
\hline 35402.1333 & $\mathrm{pg}$ & II & -22219.5 & -0.0588 & -0.0178 & -0.0195 & & (1) \\
\hline 36084.7087 & $\mathrm{pg}$ & I & -20671.0 & -0.0532 & -0.0122 & -0.0103 & & (1) \\
\hline 36361.0995 & vis & I & -20044.0 & -0.0404 & +0.0006 & +0.0038 & & (1) \\
\hline 36412.2259 & vis & I & -19928.0 & -0.0461 & -0.0052 & -0.0016 & & (1) \\
\hline 36428.7571 & vis & II & -19890.5 & -0.0447 & -0.0038 & -0.0001 & & (1) \\
\hline 36435.1503 & pg & I & -19876.0 & -0.0430 & -0.0021 & +0.0016 & & (1) \\
\hline 36731.5792 & vis & II & -19203.5 & -0.0482 & -0.0074 & -0.0023 & & (1) \\
\hline 36800.1314 & pg & I & -19048.0 & -0.0395 & +0.0012 & +0.0067 & & (1) \\
\hline 37911.364 & pg & I & -16527.0 & -0.0491 & -0.0098 & +0.0012 & & (2) \\
\hline 37911.587 & $\mathrm{pg}$ & II & -16526.5 & -0.0465 & -0.0072 & +0.0038 & & (2) \\
\hline 43347.437 & vis & II & -4194.5 & -0.0705 & -0.0525 & -0.0278 & & (3) \\
\hline 43755.442 & vis & I & -3269.0 & -0.0206 & -0.0052 & +0.0193 & & (4) \\
\hline 44438.429 & vis & II & -1719.5 & -0.0442 & -0.0334 & -0.0095 & & (5) \\
\hline 45169.5100 & pe & I & -61.0 & -0.0204 & -0.0150 & +0.0076 & +0.0035 & (6) \\
\hline 49480.5219 & pe & I & 9719.0 & +0.0243 & -0.0110 & -0.0047 & +0.0002 & (7) \\
\hline 49484.4898 & pe & I & 9728.0 & +0.0250 & -0.0104 & -0.0041 & +0.0008 & (7) \\
\hline 49486.4736 & pe & II & 9732.5 & +0.0252 & -0.0102 & -0.0039 & +0.0010 & (7) \\
\hline 50642.6965 & CCD & II & 12355.5 & +0.0450 & -0.0039 & -0.0036 & -0.0036 & (8) \\
\hline 50727.3349 & $\mathrm{CCD}$ & II & 12547.5 & +0.0509 & +0.0009 & +0.0009 & +0.0004 & (9) \\
\hline 51420.4986 & $\mathrm{CCD}$ & I & 14120.0 & +0.0657 & +0.0070 & +0.0034 & +0.0004 & (10) \\
\hline 51680.572 & pe & I & 14710.0 & +0.0705 & +0.0085 & +0.0035 & -0.0001 & (11) \\
\hline 52086.5513 & CCD & I & 15631.0 & +0.0784 & +0.0110 & +0.0040 & -0.0001 & (12) \\
\hline 52147.3827 & pe & I & 15769.0 & +0.0802 & +0.0120 & +0.0047 & +0.0006 & (11) \\
\hline 52795.5737 & pe & II & 17239.5 & +0.0833 & +0.0062 & -0.0042 & & (13) \\
\hline 52889.2492 & pe & I & 17452.0 & +0.0900 & +0.0116 & +0.0008 & -0.0024 & (14) \\
\hline 53612.3849 & pe & II & 19092.5 & +0.1028 & +0.0141 & -0.0000 & -0.0004 & (15) \\
\hline 53648.3102 & pe & I & 19174.0 & +0.1043 & +0.0150 & +0.0008 & +0.0005 & (15) \\
\hline 53851.2977 & $\mathrm{CCD}$ & II & 19634.5 & +0.1052 & +0.0130 & -0.0021 & -0.0015 & (16) \\
\hline 53943.2069 & $\mathrm{CCD}$ & I & 19843.0 & +0.1088 & +0.0152 & -0.0003 & +0.0008 & (16) \\
\hline
\end{tabular}

a(1) Tsesevitch \& Grigorevskij (1961); (2) Makarenko (1965); (3) Dietholm (1977); (4) Dietholm (1978); (5) Dietholm (1980); (6) Hoffmann (1983); (7) Hübscher et al. (1994); (8) Krobusek (1997); (9) Blättler (1998); (10) Agerer et al. (2001); (11) Agerer \& Hübscher (2002); (12) Blättler (2001); (13) Hübscher (2005); (14) Krajci (2005); (15) Hübscher et al. (2006); (16) the present paper. 
The $(\mathrm{O}-\mathrm{C})_{1}$ curve displayed in Figure 2 shows that the period change of NY Lyr is not so simple. The upward parabolic change of the $(\mathrm{O}-\mathrm{C})_{1}$ curve may indicate a continuous period increase. However, we show that only a parabolic curve can not describe the general $(\mathrm{O}-\mathrm{C})_{1}$ trend. Therefore, we consider a combination of a longterm period increase and a cyclic oscillation. A weighted least-squares solution with weights 1 to visual or photographic data and 8 to photoelectric or CCD data yields the following equation:

$$
\begin{aligned}
H J D_{\mathrm{I}_{\min }}= & 2445196.4136( \pm 0.0010) \\
& +0.44079759( \pm 0.00000024) E \\
& +8.01( \pm 0.47) \times 10^{-11} E^{2} \\
& +0.0247( \pm 0.0031) \\
& \times \sin _{\operatorname{deg}}[0.0053 E+293.7( \pm 8.7)] .
\end{aligned}
$$

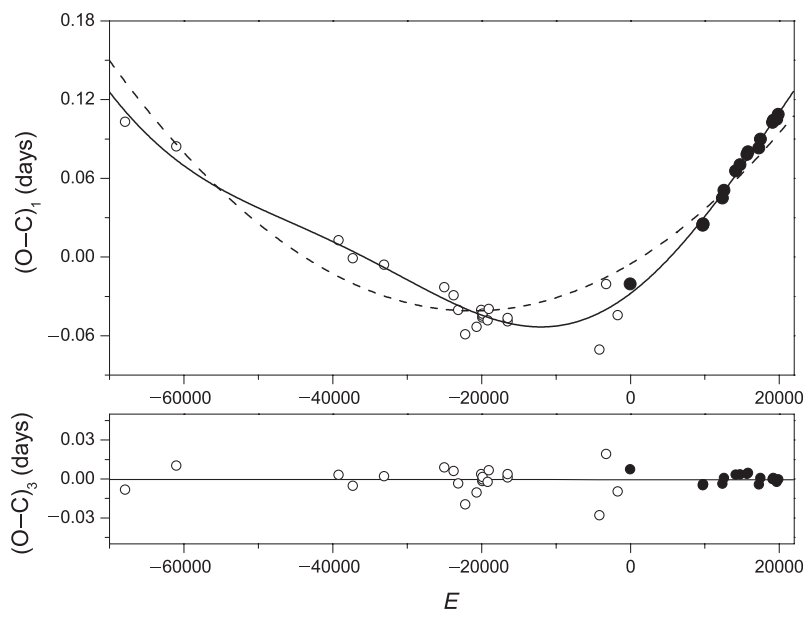

Figure 2 O-C curves of NY Lyr from the mean linear ephemeris given by Kreiner et al. (2001). Open circles refer to photographic and visual data and solid cycles to CCD and photoelectric observations. The solid line in the upper panel refers to a combination of a secular period increase and a cyclic variation. The dashed line represents the quadratic part in Equation (2). $(\mathrm{O}-\mathrm{C})_{3}$ of NY Lyr respect to the whole effect of Equation (2) are also displayed in the lower panel.

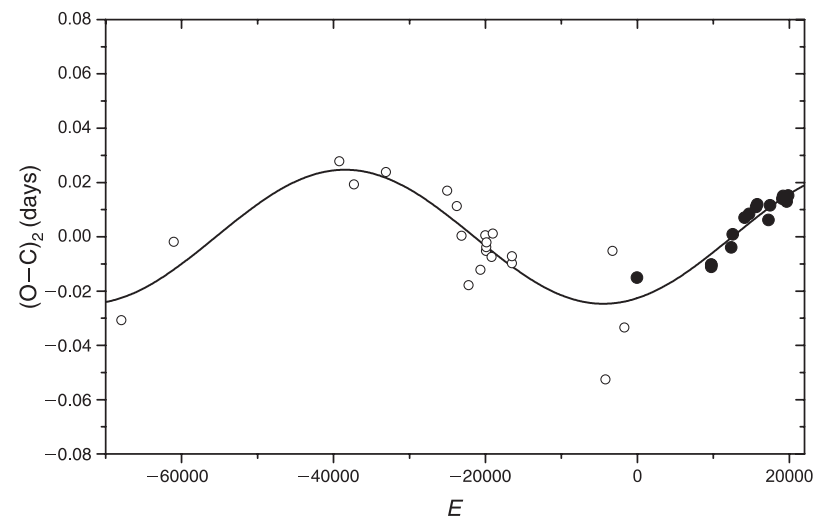

Figure $3(\mathrm{O}-\mathrm{C})_{2}$ curve of NY Lyr based on the quadratic ephemeris in Equation (4). Also given in the solid line is its fit by the sinusoidal term. Symbols are the same as in Figure 1.
By using the quadratic term, the rate of the continuous period change is derived to be $\mathrm{d} P / \mathrm{d} t=+1.33( \pm 0.08) \times$ $10^{-7} \mathrm{~d} \mathrm{yr}^{-1}$. The $(\mathrm{O}-\mathrm{C})_{3}$ residuals respect to Equation (2) are plotted in the lower panel of Figure 2. The sinusoidal term suggests a cyclic period oscillation with an amplitude of $0.0247 \mathrm{~d}$ and a period of $82.1 \mathrm{yr}$, which is more clearly seen in Figure 3 where $(\mathrm{O}-\mathrm{C})_{2}$ values based on the quadratic part in Equation (2) are displayed.

The $(\mathrm{O}-\mathrm{C})_{3}$ residuals of all photoelectric and CCD times of light minimum are plotted in Figure 4 where a small-amplitude period oscillation is apparently seen. With least-squares method, the following equation,

$$
\begin{aligned}
(\mathrm{O}-\mathrm{C})_{3}= & -0.0011( \pm 0.0002) \\
& +0.0053( \pm 0.0007) \\
& \times \sin _{\operatorname{deg}}[0.0223 E+96.6( \pm 6.3)]
\end{aligned}
$$

was determined. The equation indicates that there seems to exist another cyclic oscillation with a period of $19.4 \mathrm{yr}$ and a small amplitude of $0.0053 \mathrm{~d}$. During the computation of Equation (3), the $(\mathrm{O}-\mathrm{C})_{3}$ value of one secondary eclipse time, HJD52795.5737, was not used, because its error is larger than those of the other data points $( \pm 0.0010)$ and it shows a large scatter when compared with the general trend formed by the other data points.

However, the data in Figure 3 are only covered one cycle of the large-amplitude cyclic variation. As for the small-amplitude one, the data do not cover the whole cycle, and the first lone point in Figure 4 plays a very significant role in relation to the whole idea of the second cyclic oscillation. More times of light minimum are required in the future to ascertain the period changes presented here.

\section{Discussions and Conclusions}

Two CCD photometric times of light minimum were obtained for NY Lyr by using the 1.0-m telescope at the Yunnan Observatory in China. As in the cases of a few

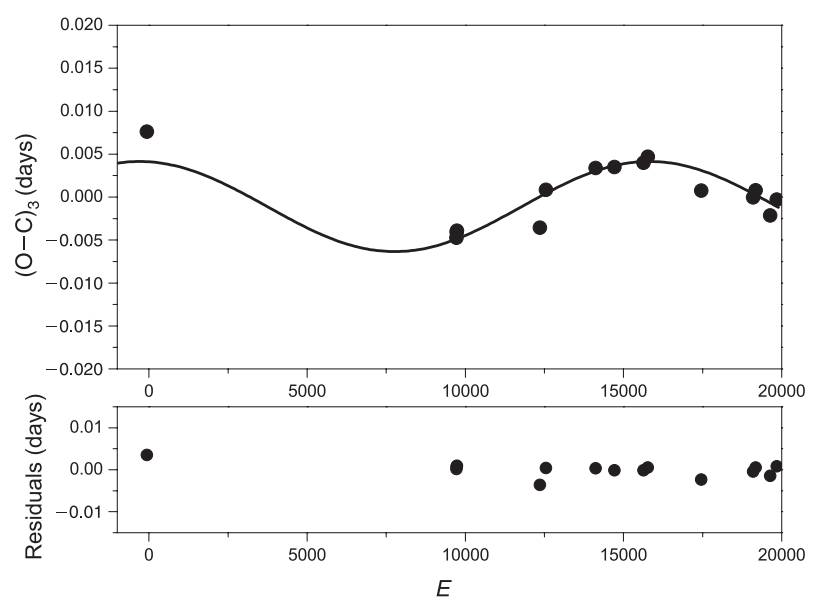

Figure $4(\mathrm{O}-\mathrm{C})_{3}$ diagram of NY Lyr formed by all photoelectric and CCD observations. A small-amplitude oscillation can be seen (solid line). Residuals after the small amplitude is removed are displayed in the lower panel. 
Table 4. Properties of period changes of four short-period W UMa-type binary stars

\begin{tabular}{lccccccc}
\hline Binaries & Period $(\mathrm{d})$ & $\mathrm{d} P / \mathrm{d} t\left(10^{-7} \mathrm{~d} \mathrm{yr}^{-1}\right)$ & $A_{3}(\mathrm{~d})$ & $P_{3}(\mathrm{yr})$ & $A_{4}(\mathrm{~d})$ & $P_{4}(\mathrm{yr})$ & Ref. $^{\mathrm{a}}$ \\
\hline AH Cnc & 0.221 & +3.99 & 0.0237 & 36.5 & 0.0035 & 7.75 & $(1)$ \\
AD Cnc & 0.360 & +4.94 & 0.0155 & 16.2 & 0.0051 & 6.60 & $(2)$ \\
FG Hya & 0.328 & -1.96 & 0.0289 & 36.4 & 0.0060 & 20.90 & $(3),(4)$ \\
NY Lyr & 0.441 & +1.33 & 0.0247 & 82.1 & 0.0053 & 19.40 & $(5)$ \\
\hline
\end{tabular}

a(1) Qian et al. (2006a); (2) Qian et al. (2007); (3) Qian \& Yang (2005); (4) Qian et al. (2006b); (5) the present authors.

W UMa-type binary systems, e.g. AH Cnc (Qian et al. 2006a), AD Cnc (Qian et al. 2007) and FG Hya (Qian \& Yang 2005; Qian et al. 2006b), the orbital period of NY Lyr displays apparently complex changes. Two periodic variations were discovered to be superimposed on a long-term continuous change. The properties of the period changes of those systems are shown in Table 4. As one can see from this table, the values of the period changes of NY Lyr are typical among the four binary stars. The recent period studies by Qian (2001a,b, 2003) showed that the period change of W UMa-type binaries may correlate with the mass ratio of the system and with the mass of the primary component. To check this conclusion, new photometric and spectroscopic observations and a careful investigation on those data are required. Moreover, the cyclic period oscillations could be explained as the light-travel time effects via the presence of additional companions. This may help to form the short-period W UMa-type system (NY Lyr) by removing angular momentum from the central system via Kozai oscillation (Kozai 1962) or a combination of Kozai cycle and tidal friction (e.g. Fabrycky \& Tremaine 2007). To check the period variations predicted here, more accurate epochs of minimum light are needed in the future.

\section{Acknowledgments}

This work was partly supported by Yunnan Natural Science Foundation (No. 2005A0059M), Chinese Natural Science Foundation (No. 10573032, No. 10573013, and No. 10433030), and by the Ministry of Science and Technology of the People's Republic of China through grant 2007CB815406. We thank Profs. Kreiner M. J. and Kim C.-H. who sent us the times of light minimum of NY Lyr. New CCD data of the binary were obtained with the 1.0-m telescope at Yunnan Observatory. The authors thank the referee for those useful comments and suggestions that help to improve the original manuscript.

\section{References}

Agerer, F., Dahm, M. \& Hubscher, J., 2001, IBVS, 5017

Agerer, F. \& Hübscher, J., 2002, IBVS, 5296

Blättler, E., 1998, BBASG, 116

Blättler, E., 2001, BBASG, 126

Dietholm, R., 1977, BBASG, 34

Dietholm, R., 1978, BBASG, 39

Dietholm, R., 1980, BBASG, 49

Fabrycky, D. \& Tremaine, S., 2007, ApJ, 669, 1298

Hoffmann, M., 1983, IBVS, 2344

Hübscher, J., 2005, IBVS, 5643

Hübscher, J., Agerer, F., Frank, P. \& Wunder, E., 1994, BAVSM, 68 Hübscher, J., Paschke, A. \& Walter, F., 2006, IBVS, 5731

Kholopov, P. N., Samus, N. N., Frolov, M. S. et al., 1985, GCVS (4th edition; Moscow: Nauka)

Kozai, Y., 1962, AJ, 67, 591

Krajci, T., 2005, IBVS, 5592

Kreiner, M. J., Kim, C.-H. \& Nha, I.-S., 2001, An Atlas of O-C Diagrams of Eclipsing Binaries (Cracow: WNAP), 1520

Krobusek, B., 1997, BBASG, 115

Makarenko, E. N., 1965, PZ, 15, 445

Morgenroth, O., 1935, AN, 256, 281

Qian, S.-B., 2001a, MNRAS, 328, 635

Qian, S.-B., 2001b, MNRAS, 328, 914

Qian, S.-B., 2003, MNRAS, 342, 1260

Qian, S.-B. \& Yang, Y.-G., 2005, MNRAS, 356, 765

Qian, S.-B., Liu, L., Soonthornthum, B., Zhu, L.-Y. \& He, J.-J., 2006a, AJ, 131, 3028

Qian, S.-B., Yang, Y.-G., Zhu, L.-Y., He, J.-J. \& Yuan, J.-Z., 2006b, Ap\&SS, 304, 25

Qian, S.-B., Yuan, J.-Z., Soonthornthum, B., Zhu, L.-Y., He, J.-J. \& Yang, Y.-G., 2007, ApJ, 671, 811

Tsesevitch, V. P. \& Grigorevskij, B., 1961, PZ, 13, 290

Yang, Y.-L. \& Li, L.-F., 1999, Publication of Yunnan Observatory, 1,32 